\title{
VARIAÇÃO ESPACIAL DO GRAU DE TROFIA E DA BIOMASSA FITOPLANCTÔNICA NO RESERVATÓRIO DE ITUPARARANGA (SÃO PAULO, BRASIL)
}

\section{SPATIAL VARIATION OF TROPHIC STATUS AND PHYTOPLANKTONIC BIOMASS IN ITUPARARANGA RESERVOIR (SÃO PAULO, BRAZIL)}

\section{Ricardo Hideo Taniwaki ${ }^{1}$, André Henrique Rosa ${ }^{2}$, Maria do Carmo Calijuri ${ }^{3}$, Viviane Moschini Carlos ${ }^{4}$}

\author{
${ }^{1}$ Biólogo, Mestrando do Programa de Pós-graduação em Engenharia Civil e \\ Ambiental - Universidade Estadual Paulista "Júlio de Mesquita Filho" (UNESP) \\ Campus Sorocaba. e-mail: rht.bio@gmail.com. \\ ${ }^{2}$ Químico, Prof. Dr. Curso de Engenharia Ambiental - Universidade Estadual Paulista \\ "Júlio de Mesquita Filho" (UNESP) Campus Sorocaba. \\ ${ }^{3}$ Bióloga, Profa. Dra. Programa de Pós-Graduação em Engenharia Hidráulica e \\ Saneamento - Universidade de São Paulo (USP) Campus São Carlos. \\ ${ }^{4}$ Bióloga, Profa. Dra. Curso de Engenharia Ambiental - Universidade Estadual \\ Paulista "Júlio de Mesquita Filho" (UNESP) Campus Sorocaba.
}

\section{RESUMO}

Atualmente os reservatórios brasileiros vêm sofrendo grandes pressões devido à crescente demanda por água para diversos fins, sendo a eutrofização uns dos maiores problemas causados pelo homem. O reservatório de Itupararanga fornece serviços ambientais para mais de um milhão de pessoas e é um dos principais manancias da bacia do rio Sorocaba e médio Tietê. Desta forma, o presente trabalho teve como objetivo verificar a variabilidade espacial do grau de trofia e da biomassa fitoplanctônica do reservatório, assim como as concentrações de nutrientes e também verificar seu enquadramento conforme a resolução CONAMA 357/2005 (BRASIL, 2005). Para isto foi realizada uma coleta da água superficial em seis estações amostrais e medidas de $\mathrm{pH}$, condutividade elétrica, oxigênio dissolvido, temperatura da água, transparência da água, fósforo total, fósforo total dissolvido, nitrito, nitrato, concentrações de clorofila-a e no sedimento foi realizada análises granulométricas. Os resultados indicaram que não houve variação espacial do grau de trofia do reservatório de Itupararanga, porém houve variação espacial das concentrações de nutrientes e clorofila-a, corroborando com modelos teóricos de compartimentalização de reservatórios.

Palavras-chave: Reservatório. Itupararanga. Trofia. Nutrientes. CONAMA

\footnotetext{
ABSTRACT

Nowadays the Brazilian reservoirs has suffered great pressure due to increasing demand for water for diverse purposes, being one of the largest eutrophication problems caused by man. The Itupararanga reservoir provides environmental services to more than one million people and is one
} 
of the main sources of the Sorocaba River basin and middle Tietê. Thus this study aimed to determine the spatial variability of the trophic level and phytoplankton biomass in the reservoir, as well as the concentrations of nutrients and also check your environment according to CONAMA 357/2005 (BRASIL, 2005). For this, a collection that was conducted in six sampling stations and the measures of $\mathrm{pH}$, conductivity, dissolved oxygen, water temperature, water transparency, total phosphorus, total phosphorus, dissolved nitrite, nitrate, chlorophyll-a and sediment size analysis . The results indicated that there was no spatial variation of the degree of trophy in the Itupararanga reservoir, but there was spatial variation of nutrient concentrations and chlorophyll-a, corroborating theoretical models of reservoir compartmentalization.

Keywords: Reservoir. Itupararanga. Trophic status. Nutrient. CONAMA

\section{INTRODUÇÃO}

Reservatórios são ecossistemas artificiais que trazem benefícios e prejuízos ao ecossistema e à sociedade. Se não forem bem gerenciados, podem gerar diversos problemas, entre elas a eutrofização, que está entre os maiores impactos causados pelo homem nos reservatórios brasileiros (FIGUEIRÊDO et al. 2007; TUNDISI e MATSUMURA-TUNDISI, 2008).

A falta de saneamento básico, captação e tratamento de efluentes domésticos e industriais podem contribuir com o aumento da concentração de nutrientes nos reservatórios, eutrofizando este ecossistema. Uma das conseqüências desse processo é a floração de cianobactérias, que podem causar graves problemas à comunidade aquática e aos que usufruem do abastecimento de água potável (BRAGA et al. 2005).

O reservatório de Itupararanga é um dos principais mananciais de água potável da região da bacia do Alto Tietê e fornece serviços ambientais para milhões de pessoas (Figura 1). Foi construído com o intuito de geração de energia elétrica e com a crescente demanda por água potável, passou a servir também como fonte de água potável para a região.

Com a crescente expansão industrial e agrícola da bacia do Alto Tietê, o reservatório de Itupararanga tem sofrido diversas pressões ambientais e com isso, a qualidade de suas águas está comprometida.

Esse trabalho pretende esclarecer a seguinte hipótese: Existe variação espacial no grau de trofia e na biomassa fitoplanctônica no reservatório de Itupararanga? Também foi analisado se as concentrações de nutrientes e clorofila-a estão em conformidade com a Resolução CONAMA 357 de 2005, que dispõe sobre a classificação dos corpos de água. O reservatório de Itupararanga foi considerado como classe 2, segundo o artigo 42 da Resolução 357/2005, que propõe: "enquanto não aprovados os respectivos enquadramentos, as águas doces serão consideradas como classe 2". (BRASIL, 2005). 


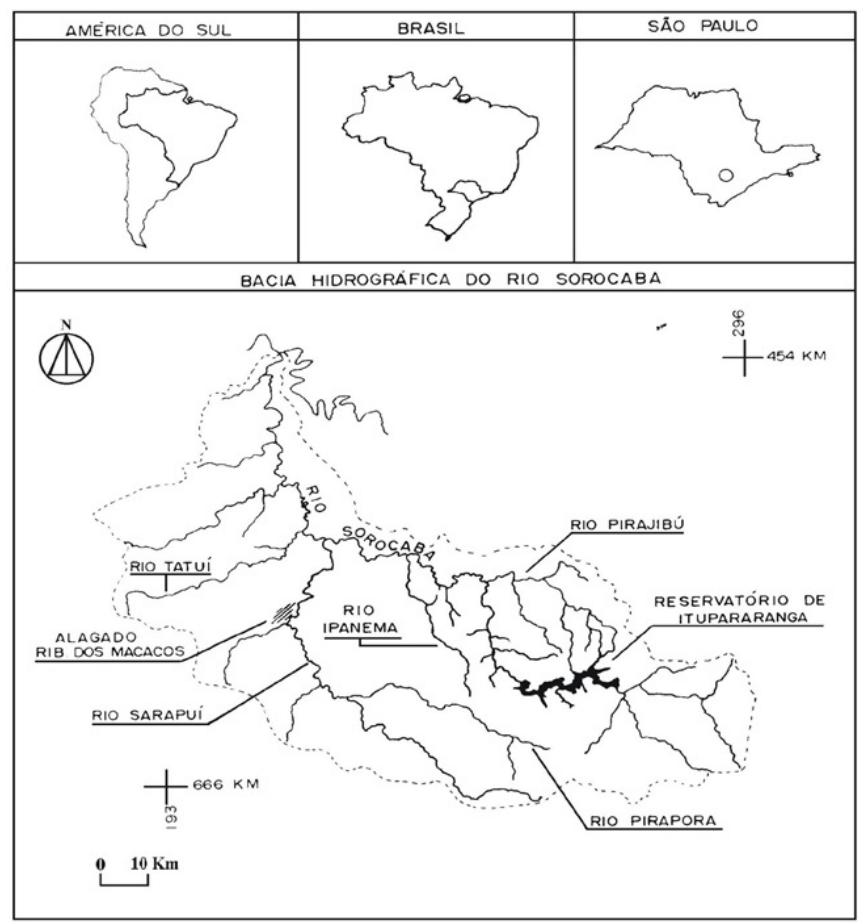

Figura 1. Localização da bacia hidrográfica do rio Sorocaba e do reservatório de Itupararanga (Smith \& Petrere Jr. 2000).

\section{MATERIAL E MÉTODOS}

Foi realizada uma coleta em setembro do ano de 2010 da água superficial em 6 estações amostrais ao longo do reservatório de Itupararanga, escolhidas com o intuito de caracterizar o gradiente vertical do grau de trofia e da biomassa fitoplanctônica (Figura 2, Tabela 1).

A estação amostral 1 está localizada na entrada dos rios Sorocabuçu e Sorocamirim, formadores do reservatório (Figura 2, Tabela 1). As estações amostrais 2, 4, 5 e 6 estão localizadas na calha central do reservatório, em sentido montante-jusante, e a estação amostral 3 está localizada na desembocadura do Córrego do Campo Verde (Figura 2, Tabela 1).

No local, foi medida a transparência da água através do disco de Secchi, a temperatura da água, o $\mathrm{pH}$ e a condutividade elétrica $\left(\mu \mathrm{S} . \mathrm{cm}^{-1}\right)$ com auxílio da sonda YSI modelo 63-50 e oxigênio dissolvido através da sonda YSI modelo 55-12.

Em cada estação amostral foram coletadas amostras de água superficial para quantificação de fósforo total (APHA, 2005), fosfato total dissolvido (APHA, 2005), nitrito (MACKERETH et al. 1978), nitrato (APHA, 2005) e clorofila-a (NUSH, 1980). Também foi coletado sedimento para análise de granulometria, seguindo o método de béqueres proposto por Piper (1947) e modificado por Meguro (2000).

A partir dos dados físicos, químicos e biológicos coletados, foi realizada a análise de correlação linear de Pearson a fim de verificar as possíveis relações entre as variáveis, análise de cluster, com o método de Ward's e distâncias euclideanas, com o intuito de verificar os possíveis agrupamentos entre as estações amostrais e assim a heterogeneidade espacial. Também foi aplicada a análise de componentes principais (ACP) com a finalidade de evidenciar e hierarquizar os fatores responsáveis pela variância da biomassa fitoplanctônica tendo sido utilizado para todas as análises o programa computacional Statística 8.0 da Statsoft Inc. 


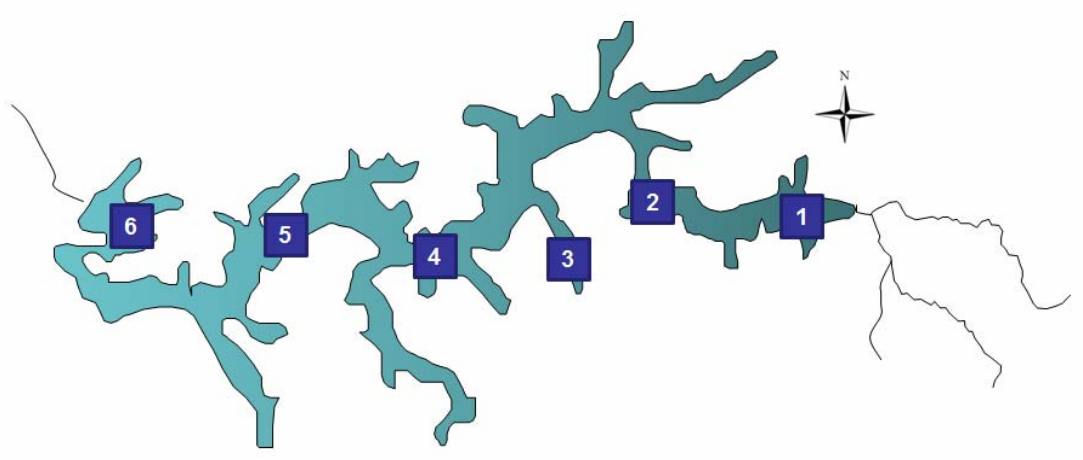

Figura 2. Estações amostrais no reservatório de Itupararanga.

\section{RESULTADOS E DISCUSSÃO}

As profundidades das estações amostrais variaram de 4,3 a 16 m, sendo a estação 4 a que apresentou maior profundidade. A transparência da água, medida através do disco de Secchi variou de 0,8 (estação amostral 3) a 1,4 m (estação amostral 4). A temperatura da água variou de 18,9 a $20,9^{\circ} \mathrm{C}$ e a condutividade elétrica variou de 31,8 (estação amostral 3) a $60,2 \mu \mathrm{S} \cdot \mathrm{cm}^{-1}$ (estação amostral 5) (Tabela 1).

$\mathrm{O}$ pH da água mostrou-se ligeiramente ácido na estação amostral $1(6,1)$ e $2(6,6)$, sendo que as demais estações apresentaram valores de $\mathrm{pH}$ variando de 7,3 a 7,6. Foi possível observar que os valores de $\mathrm{pH}$ aumentaram gradativamente no sentido montante - jusante.

Todas as estações amostrais apresentaram elevados valores de oxigênio dissolvido (variando entre 8,3 mg. $\mathrm{L}^{-1}$ a 9,2 mg.. $\mathrm{L}^{-1}$ ), sendo os maiores valores na zona lacustre do reservatório (Tabela 1).

As concentrações de fósforo total variaram de $12,1 \mathrm{mg} . \mathrm{L}^{-1}$ a $68,5 \mathrm{mg} . \mathrm{L}^{-1}$ (estação amostral 6). Em um recente estudo realizado por Miwa et al. (2011) no reservatório de Itupararanga durante o ano de 2010, foram encontradas concentrações similares a deste estudo. As concentrações de nutrientes dissolvidos variaram de $0,03 \mathrm{mg} . \mathrm{L}^{-1}$ a $0,505 \mathrm{mg} . \mathrm{L}^{-1}$ para o fosfato total dissolvido, de 0,35 mg. $\mathrm{L}^{-1}$ a $0,770 \mathrm{mg} . \mathrm{L}^{-1}$ para nitrato e abaixo do limite de detecção do método a $0,0036 \mathrm{mg} . \mathrm{L}^{-1}$ para o nitrito (Tabela 2).

Tabela 1 - Coordenadas e variáveis físicas e químicas obtidas durante o estudo.

\begin{tabular}{cccccccc}
\hline $\begin{array}{c}\text { Estação } \\
\text { amostral }\end{array}$ & $\begin{array}{c}\text { Coordenadas (UTM } \\
\mathbf{2 3 K})\end{array}$ & $\begin{array}{c}\text { Secchi } \\
(\mathbf{m})\end{array}$ & $\begin{array}{c}\text { Prof. } \\
(\mathbf{m})\end{array}$ & $\begin{array}{c}\text { Temp. } \\
\text { água } \\
\left({ }^{\mathbf{0}} \mathbf{C}\right)\end{array}$ & $\mathbf{p H}$ & $\begin{array}{c}\text { OD } \\
\left(\mathbf{m g . L}^{\mathbf{- 1}}\right)\end{array}$ & $\begin{array}{c}\text { Cond. } \\
\text { Elétrica } \\
\left(\boldsymbol{\mu} \mathbf{S}_{\text {.cm }} \mathbf{- 1}\right)\end{array}$ \\
\hline 1 & $272261 / 7385990$ & 0,80 & 6,3 & 18,9 & 6,1 & 8,9 & $*$ \\
2 & $268684 / 7388890$ & 1,20 & 5,0 & 19,6 & 6,6 & 8,7 & $*$ \\
3 & $266997 / 7385061$ & 0,48 & 0,5 & 20,2 & 6,9 & 8,3 & 31,8 \\
4 & $261411 / 7386441$ & 1,40 & 16,0 & 19,0 & 7,3 & 8,6 & 62,0 \\
5 & $259465 / 7383798$ & 1,00 & 5,4 & 20,9 & 7,3 & 9,0 & 62,1 \\
6 & $255738 / 7385353$ & 1,30 & 4,3 & 19,5 & 7,6 & 9,2 & 60,0 \\
\hline
\end{tabular}

Prof- profundidade; OD- oxigênio dissolvido. * medidas não obtidas devido a problemas técnicos na sonda

As concentrações de clorofila-a variaram de 14,2 $\mu \mathrm{g} . \mathrm{L}^{-1}$ (estação amostral 5) a 37,4 $\mu \mathrm{g} . \mathrm{L}^{-1}$ (estação amostral 1). A estação 1 está localizada à montante do reservatório, próximo à entrada dos principais rios formadores do reservatório (rio Sorocabuçu e rio Sorocamirim) (Tabela 2). Miwa et al. (2011) encontrou valores similares de clorofila-a no reservatório de Itupararanga durante o ano de 2010. 
Tabela 2 - Concentrações de nutrientes e clorofila-a quantificadas nas estações amostrais durante o estudo.

\begin{tabular}{|c|c|c|c|c|c|}
\hline $\begin{array}{c}\text { Estação } \\
\text { amostral }\end{array}$ & $\begin{array}{c}\text { Chl-a } \\
\left(\mu \mathrm{g} . \mathrm{L}^{-1}\right)\end{array}$ & $\begin{array}{c}\text { PT } \\
\left(\mu \mathrm{g} . \mathrm{L}^{-1}\right) \\
\end{array}$ & $\begin{array}{c}\mathrm{P}^{-\mathrm{PO}_{4}} \\
\left(\mathrm{mg} . \mathrm{L}^{-1}\right)\end{array}$ & $\begin{array}{c}\mathrm{N}-\mathrm{NO}_{3} \\
\left(\mathrm{mg} . \mathrm{L}^{-1}\right)\end{array}$ & $\begin{array}{c}\mathrm{N}-\mathrm{NO}_{2} \\
\left(\mathrm{mg} . \mathrm{L}^{-1}\right)\end{array}$ \\
\hline 1 & 37,4 & $*$ & 0,033 & 0,770 & 0,0036 \\
\hline 2 & 20,0 & 12,1 & 0,150 & 0,360 & 0,0008 \\
\hline 3 & 21,4 & $*$ & 0,498 & 0,410 & 0,0001 \\
\hline 4 & 15,6 & 56,1 & 0,266 & 0,440 & 0,0001 \\
\hline 5 & 14,2 & 31,7 & 0,505 & 0,350 & * \\
\hline 6 & 15,4 & 68,5 & 0,299 & 0,350 & $*$ \\
\hline
\end{tabular}

* Abaixo do limite de detecção pelo método empregado.

Em relação à análise granulométrica do sedimento, houve um grande variação entre as estações amostrais, porém todas as estações apresentaram-se com altas concentrações de matéria orgânica. As maiores concentrações foram encontradas nas estações amostrais 1, 2 e 3 próximas à entrada dos principais formadores do reservatório (Tabela 3). Segundo Kimmel et al. (1990), há predominância de matéria orgânica autóctone nas regiões ainda influenciadas pelos rios formadores.

De uma forma geral, as concentrações de areia no sedimento diminuíram no sentido jusantemontante e as concentrações de silte aumentaram no sentido jusante-montante devido a maior mobilidade deste elemento junto ao fluxo da água. De acordo com Henry et al. (1998) a sedimentação é decorrente da redução acentuada da velocidade na zona de transição entre rio e represa.

Através da análise de cluster, considerando as variáveis da água superficial (transparência da água, profundidade, temperatura, $\mathrm{pH}$, oxigênio dissolvido, sólidos suspensos totais, clorofila-a, fósforo total) e do sedimento (argila, areia, silte e matéria orgânica), foi possível observar o agrupamento das estações amostrais 1, 2 e 3, consideradas como a zona de rio, com maior quantidade de matéria orgânica no sedimento e maiores concentrações de clorofila-a e nutrientes na água. As estações amostrais 4 e 5 como a zona de transição e a estação amostral 6 como a zona lacustre, com menores concentrações de matéria orgânica no sedimento, menores concentrações de clorofila-a na água e maiores concentrações de oxigênio dissolvido na água (Figura 3).

Tabela 3 - Porcentagens das frações de argila, silte, areia e matéria orgânica nas estações amostrais.

\begin{tabular}{ccccc}
$\begin{array}{c}\text { Estações } \\
\text { amostrais }\end{array}$ & $\begin{array}{c}\text { Granulometria } \\
\text { Areia } \\
\text { \% }\end{array}$ & $\begin{array}{c}\text { Argila } \\
\text { \% }\end{array}$ & $\begin{array}{c}\text { Silte } \\
\text { \% }\end{array}$ & Mat. Org. \\
\hline 1 & 58,4 & 23,7 & 5,1 & 89,29 \\
2 & 54,7 & 40,7 & 9,2 & 89,24 \\
3 & 37,1 & 28,5 & 13,3 & 88,04 \\
4 & 39,5 & 21,2 & 21,6 & 82,99 \\
5 & 28,4 & 47,7 & 12,2 & 85,87 \\
6 & 81,5 & 4,9 & 9,0 & 87,44 \\
\hline
\end{tabular}

Este resultado evidencia a compartimentalização do reservatório segundo a teoria de Thornton et al. (1990). Segundo estes autores, os reservatórios podem ser divididos em 3 regiões, onde a primeira sofre influências dos rios, tendo altas concentrações de material particulado e nutrientes, a segunda região tem características intermediárias de rios e lagos e a terceira região possui características de lagos, com menores concentrações de sólidos suspensos na água, menores concentrações de nutrientes e características relativamente oligotróficas. 


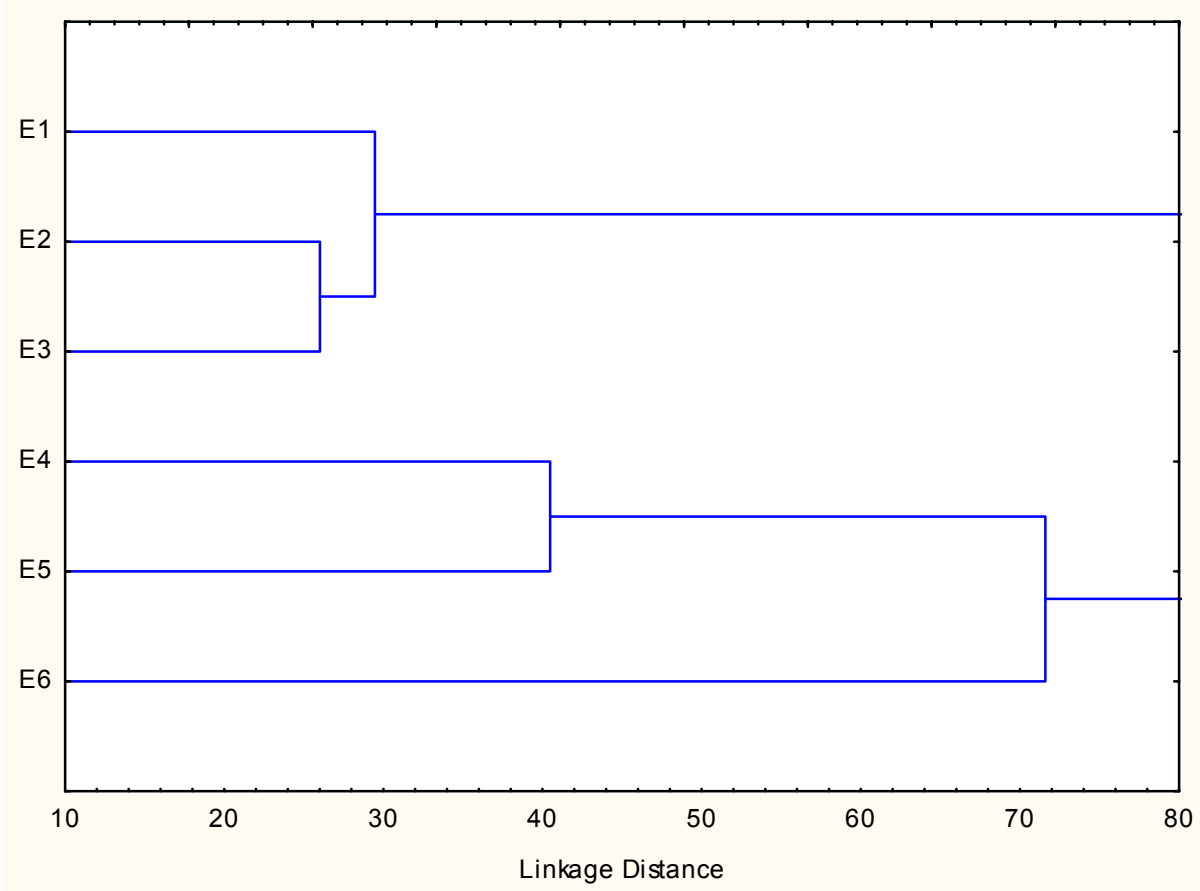

Figura 3. Análise de cluster, descrevendo o agrupamento entre as estações amostrais no reservatório de Itupararanga. (E1- Estação amostral 1; E2 - Estação amostral 2; E3 - Estação amostral 3; E4 - Estação amostral 4; E5 - Estação amostral 5; E6 - Estação amostral 6).

A análise de correlação linear de Pearson evidenciou correlação fortemente negativa entre a transparência da água (disco de Secchi) e o material particulado em suspensão (Tabela 4), evidenciando a influência da biomassa fitoplanctônica e de outros sólidos suspensos presentes na água na distribuição da zona fótica no reservatório, corroborando a teoria de Thornton et al. (1990).

A profundidade teve correlação fortemente negativa com as concentrações de matéria orgânica presente no sedimento, sendo que, de uma forma geral, as maiores concentrações foram encontradas nas zonas a montante do reservatório e diminuindo à jusante. Esse fato pode estar associado á redução das concentrações de biomassa fitoplanctônica neste mesmo sentido, e consequentemente diminuindo a deposição deste material no sedimento (Tabela 4).

Com o intuito de verificar as relações da biomassa fitoplanctônica (expressa em clorofila-a) e as variáveis físicas e químicas da água, foi realizada a análise de componentes principais, na qual agrupou a clorofila-a com os valores de oxigênio dissolvido, $\mathrm{pH}$, condutividade, fósforo total e transparência da água, evidenciando a correlação direta entre estes fatores (Figura 4). De acordo com Reynolds (2006), o fósforo é um fator limitante para a ocorrência dos produtores primários e, desta forma, a disponibilidade do fósforo propicia o crescimento e aumento do fitoplâncton, alterando as concentrações de oxigênio dissolvido, o pH e também a transparência da água. 
Tabela 4 - Valores significativos das correlações lineares de Pearson entre as variáveis físicas, químicas e biológicas.

\begin{tabular}{lcccc}
\hline & zDS & Prof & Cond & MPS \\
\hline zDS & & & & \\
Prof & & & & \\
Cond & & & & \\
MPS & $-0,99$ & & $-0,95$ & \\
Chl-a & & & $-0,98$ & \\
PT & 0,96 & & & $-0,95$ \\
$\mathrm{NO}_{3}{ }^{-}$ & & & & \\
$\mathrm{NO}_{2}{ }^{-}$ & & & & \\
$\mathrm{MO}^{-}$ & & $-0,98$ & & \\
\hline
\end{tabular}

* (zDS- Transparência da água medida através do disco de Secchi; Prof- profundidade da estação amostral; Cond- condutividade elétrica; MPS- material particulado suspenso; Chl-a- clorofila-a, PT- fósforo total; $\mathrm{NO}_{3}$ - nitrato; $\mathrm{NO}_{2}{ }^{-}$nitrito e MO- matéria orgânica do sedimento).

Em relação ao grau de trofia do reservatório, calculado pelo método proposto por Lamparelli (2004) através dos valores de clorofila-a, a estação amostral 1, localizada a montante do reservatório na entrada dos rios Sorocabuçu e Sorocamirim encontra-se em condições hipereutróficas, representando o maior potencial de prejuízos decorrentes da produtividade algácea e o maior potencial para produtividade de algas e plantas.

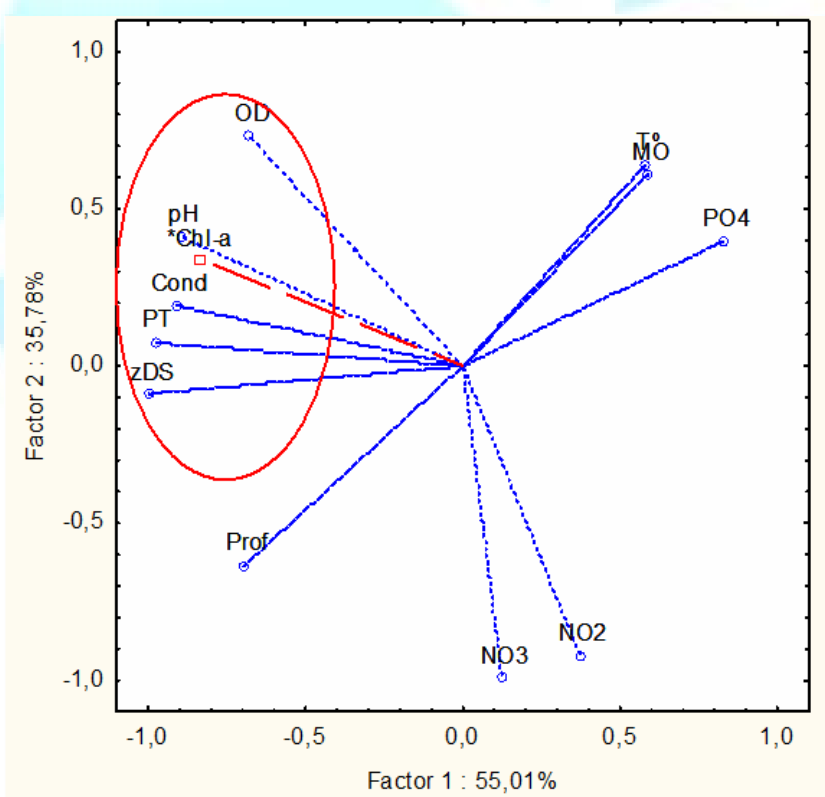

Figura 4. Relações entre a biomassa fitoplanctônica e parâmetros ambientais referentes aos dois primeiros componentes principais no reservatório de Itupararanga. (OD- oxigênio dissolvido; Chl-a- clorofila-a; CondCondutividade, PT- fósforo total; zDS- transparência da água medida através do disco de Secchi; Profprofundidade; NO3- nitrato; NO2- nitrito, $\mathrm{PO} 4$ - fosfato total dissolvido, MO- matéria orgânica do sedimento e $\mathrm{T}^{\mathrm{o}}$ - temperatura da água). 
As outras estações amostrais foram classificadas como supereutróficas pelo índice. Esta classificação sugere que essas estações amostrais correm sérios riscos referentes aos prejuízos decorrentes da produtividade algácea e grande potencial para produtividade de algas e plantas.

Tabela 5 - Valores calculados do Índice de Estado Trófico (IET) proposto por Lamparelli (2004).

\begin{tabular}{ccc}
$\begin{array}{c}\text { Estação } \\
\text { amostral }\end{array}$ & IET (CI) & Categoria \\
\hline 1 & 68,6 & Hipereutrófico \\
2 & 65,5 & Supereutrófico \\
3 & 65,8 & Supereutrófico \\
4 & 64,3 & Supereutrófico \\
5 & 63,8 & Supereutrófico \\
6 & 64,2 & Supereutrófico \\
\hline
\end{tabular}

De acordo com Figueirêdo et al. (2007), a urbanização, a agropecuária e o desmatamento aumentam a carga de nutrientes nos reservatórios, contribuindo com o processo de eutrofização. Segundo Beu et al. 2011, o entorno do reservatório de Itupararanga é ocupado em 31,03\% de áreas de atividades rurais, sendo que a cobertura por vegetação ripária em sua margem esquerda (sentido barragem) é escassa.

Em relação à conformidade com os padrões da resolução CONAMA 357/2005, os valores de oxigênio dissolvido e $\mathrm{pH}$ estão dentro das condições exigidas para a classe $2 \mathrm{em}$ todas as estações amostrais ( $>5 \mathrm{mg} . \mathrm{L}^{-1}$ para OD e $\mathrm{pH}$ de 6-9), as concentrações de clorofila-a estão dentro das condições exceto a estação 1 que ultrapassou a concentração máxima permitida para a classe 2 de $30 \mu \mathrm{g} \cdot \mathrm{L}^{-1}$. (BRASIL, 2005)

As concentrações de fósforo total excederam os valores padrões para a classe 2 em todas as estações amostrais (concentração máxima permitida de $0,030 \mathrm{mg} . \mathrm{L}^{-1}$ ), trazendo problemas relativos à qualidade da água através do aumento da biomassa algal e de outros produtores primários.

As concentrações de nitrato e nitrito estão dentro das concentrações máximas permitidas (10 mg.L ${ }^{-1}$ e 1 mg. $L^{-1}$ respectivamente) para a classe 2.

\section{CONCLUSÕES}

Através do presente estudo, foi possível observar que houve variação espacial no grau de trofia no reservatório de Itupararanga em setembro de 2010, sendo a estação amostral 1 considerada hipereutrófica e as outras estações amostrais consideradas supereutróficas. Foi possível observar a variação da biomassa fitoplanctônica no sentido rio-barragem, evidenciando a compartimentalização do reservatório, como sugerido por Thornton et al. (1990) e também encontrado por Miwa et al. (2011). Em relação aos valores de referência exigidos para a classe 2 do CONAMA 357/2005 (BRASIL, 2005), na qual o reservatório está enquadrado, as concentrações de fósforo total estão acima do permitido em todas as estações amostrais. A concentração de clorofilaa excedeu somente na estação 1 (à montante do reservatório).

Com isso, sugere-se manejo e monitoramento constante no reservatório de Itupararanga, além da recomposição da vegetação ciliar em sua margem esquerda (sentido barragem) visando 
garantir a proteção à vida aquática e à sustentabilidade deste recurso hídrico, tendo em vista as condições preocupantes relativas ao grau de trofia desse reservatório durante o período de estudo.

\section{AGRADECIMENTOS}

Agradecemos à FAPESP (Proc. 08/55636-9) e a CAPES pela bolsa de mestrado concedida ao primeiro autor do trabalho.

\section{REFERÊNCIAS}

APHA. Standard methods for the examination of water and waste-water. Washington: Am. Public. Health Assoc., 1985. 1268p.

BEU, S.E.; MISATO, M.T.; HAHN, C.M. APA de Itupararanga. In: BEU, S.E.; SANTOS, A.C.A.; CASALI,S. Biodiversidade na APA Itupararanga: Condições atuais e perspectivas futuras. São Paulo: SMA/FF/UFSCar/CCR-Via Oeste, 2011.

BRAGA, B., HESPANHOL, I., CONEJO, J. G.; LOTUFO. Introdução à Engenharia Ambiental (2a. Ed). São Paulo: Prentice Hall. 2005.

BRASIL. Ministério do Meio Ambiente. Conselho Nacional do Meio Ambiente. Resoluções do CONAMA $n^{\circ}$. 357. (5ed). Brasília, DF. SEMA, 2005. 23 p.

FIGUEIRÊDO, M.C.B.; TEIXEIRA, A.S.; ARAÚJO, L.F.P.; ROSA, M.F.; PAULINO, W.D.; MOTA, S.; ARAÚJO, J.C. Avaliação da vulnerabilidade ambiental de reservatórios à eutrofização. Eng. Sanit. Ambient., v. 12, n. 4, 2007.

HENRY R., NUNES M. A., MITSUKA P. M., LIMA N. DE, CASANOVA S. M. C.. Variação espacial e temporal da produtividade primária pelo fitoplâncton na represa de Jurumirim (Rio Paranapanema, SP). Rev. Bras. Biol. v. 58, n.4, P. 571-590, 1998.

KIMMEL, B.L., LIND, O.T. PAULSON, L.J. Reservoir primary production. In: THORNTON, K.W., PAYNE, F.E.; KIMMEL, B.L. Reservoir limnology, Ecological perspectives. United States: John Wiley \& Sons Inc. 1990.

LAMPARELLI , M. C. Grau de trofia em corpos d'água do estado de São Paulo: avaliação dos métodos de monitoramento. 2004. 235 f. Tese (Doutorado em Ciências) - Departamento de Ecologia, Universidade de São Paulo- USP, 2004.

MACKERETH, F. J. H., HERON, J.; TALLING, J. F. Water analysis: some revised methods for limnologists. Freshwater Biological Association. n.36, 1978.

MIWA, A.C.P.; BOTTINO, F.; SANTOS, A.C.A.; CALIJURI, M.C. Limnologia no reservatório de Itupararanga. In: BEU, S.E.; SANTOS, A.C.A.; CASALI,S. Biodiversidade na APA Itupararanga: Condições atuais e perspectivas futuras. São Paulo: SMA/FF/UFSCar/CCR-Via Oeste, 2011. 
NUSH, E. A. Comparison of diferent methods for clorophyll and phaeopigment determination. Arch. Hydrobiol. Bech. Stuttgart. v. 14, P.14-36. 1980.

REYNOLDS, C. Ecology of phytoplankton. New York: Cambridge University Press, 2006.

SMITH, W. S.; PETRERE JR., M. Caracterização limnológica da bacia de drenagem do Rio Sorocaba, São Paulo, Brasil. Acta Limnologia Brasiliensia. v.12, p. 15-27, 2000.

STATSOFT, INC. STATISTICA (data analysis software system), version 8.0. www.statsoft.com. 2007.

THORNTON, K.W., PAYNE, F.E.; KIMMEL, B.L. Reservoir limnology, Ecological perspectives. United States: John Wiley \& Sons Inc. 1990.

TUNDISI, J.G.; MATSUMURA-TUNDISI, T. Limnologia. São Paulo: Oficina de Textos, 2008. PIPER, C.S. Soil and plant analysis. New York: Interscience. 1947. 368p.

MEGURO, M. Métodos em ecologia. São Paulo: USP. 2000. 117p. 